\title{
Familias y procesos que reportan el logro de objetivos terapéuticos en el Centro de Familia de la Universidad Pontificia Bolivariana
}

\section{Isabel Cristina Bernal Vélez}

Magister en Terapia Familiar de la UPB, especialista en Familia, especialista en Terapia Familiar Sistémica, coordinadora del Área de Asistencia del Centro de Familia de la UPB, docente asociada de la Especialización en Familia y a la Maestría en Terapia Familiar de la UPB, investigadora del Grupo de Investigación en Familia UPB.Correo: isabel.bernal@upb.edu.co

\section{Piedad Estrada Arango}

Magister en Terapia Familiar de la UPB, especialista en Familia de la UPB, docente titular de la Especialización en Familia y de la Maestría en Terapia Familiar de la UPB, investigadora del Grupo de Investigación en Familia de la UPB. Correo: pestrada1608@une.net.co

\section{Marta Aída Palacio Correa}

Aprobado: 30 de julio de 2020

Recibido: 24 de enero de 2020 DOI: http://dx.doi.org/10.18566/rfts.v35n35.a02
Magister en Epidemiología de la Universidad de Antioquia, especialista en Economía y Negocios Internacionales, docente titular e investigadora del Grupo de Investigación en Familia de la UPB.

Correo:marta.palacio@upb.edu.co 


\section{Resumen}

Este artículo presenta resultados derivados de una investigación descriptiva de diseño cuantitativo, cuyo propósito principal fue identificar el perfil de las familias que terminan procesos de terapia familiar con objetivos cumplidos en el Centro de Familia de la UPB. Para lograrlo, se seleccionaron entre 48.759 historias clínicas, aquellas familias que terminaron el proceso terapéutico con logros de objetivos según la percepción del profesional y la opinión de las familias consultantes, resultó un total de 2.520 procesos terapéuticos. Se incluyeron como variables de análisis: formas de organización familiar, etapas del ciclo vital, problemáticas consultadas según percepción del profesional, motivos de consulta expresados por las familias, número de citas atendidas, tiempo de duración del proceso, entre otras. Los hallazgos indican que las familias nucleares, en etapa de escolaridad y adolescencia, están altamente representadas en la población objeto de estudio y logran adquirir habilidades que les permiten fortalecer el cumplimiento de funciones relacionadas con los motivos de consulta prioritarios, como casos de obstáculos en el ejercicio de la autoridad, pautas de crianza, normas, sanciones, relación de dependencia del hijo hacia el padre, madre y viceversa, y dificultades en la comunicación. El alcance de estos objetivos es logrado por la familia en un tiempo de seis meses y con una asistencia de entre cinco y más citas. Lo anterior permite concluir que es posible lograr resultados en procesos de corta duración.

\section{Palabras clave}

Ciclo vital familiar, Logro de objetivos, Familia, Formas de organización familiar, Terapia familiar, Procesos terapéuticos. 


\section{Abstract}

This article is derived from a research that selected 48,759 medical histories on families who finished the therapeutical procedure with accomplishments such as their professional perception and the consultant family's opinion, resulting in a total of 2,520 therapeutical procedures with these characteristics, giving out the following variables: ways of family organization, steps of their vital cycle, problems consulted according to a professional perception and certain motives for a consult expressed by families, the number of attended families, the duration of the process, and some other aspects. This is a descriptive quantitative study, whose general objective is to identify the profile of the families that finish therapeutical processes with their objectives and goals accomplished in the Family Centre of the UPB. The main results indicate that nuclear family, in the stage of schoolarity and adolescence, are highly represented in this population and they succeed on adquiring abilities that allow them to strengthen the accomplishment of functions related to the consult's overall motive, as are the obstacles on the exercise of authority, bases of how to raise an infant, rules, punishments, infant's relationship with the father, mather and vice versa, and the difficulties on communication. The reaching of this goals is accomplished by each family over a time of six months and with five or six appointments.

Keywords

Vital family cycle, Therapeutical success, Family, Ways of family organization, Family therapy. 


\section{Introducción}

La terapia familiar es un proceso que se convierte en factor facilitador de cambio para quienes la solicitan. Transitar por ella puede ser algo que genera incertidumbre a los consultantes, ya que moviliza, revela, examina y trae al presente acontecimientos que resultan dolorosos, los cuales les lleva a sentir desazón y malestar al verse enfrentados a reflexionar sobre las situaciones que los convocan. Reconocer las condiciones por resolver y afrontarlas, puede contribuir a que dicha dificultad se convierta en un posibilitador de crecimiento y de construcción de nuevas realidades.

Desde el Centro de Familia de la Universidad Pontificia Bolivariana, terapeutas familiares, profesionales en entrenamiento, psicólogos y personas relacionadas con el estudio y la intervención en los temas relacionados con la salud mental, se encuentran con que la mayoría de las veces los estudios enfatizan el discurso del déficit, pero es de interés resaltar también las posibilidades y logros en los procesos de ayuda. Algo que nos lleva a plantear el siguiente interrogante: ¿Cuáles son las características de las familias que culminan los procesos terapéuticos alcanzando los objetivos trazados?

Este texto presenta resultados de una investigación con 2.520 procesos de familias atendidas en el Centro de Familia de la Universidad Pontificia Bolivariana entre los años 1999 y 2013, periodo en el cual se contaba con el registro de la totalidad de historias clínicas. Nuestro propósito principal pasa por responder el interrogante anterior y conocer las características sociodemográficas de las familias, así como la intervención recibida en los procesos terapéuticos que culminan con el logro de objetivos, según la percepción del terapeuta, quien consigna esta información en los registros institucionales. Se presentan algunos datos relacionados con el grupo familiar, características del proceso terapéutico y motivos de consulta expresados por los consultantes. Del mismo modo, se incluye en el artículo un aspecto referente a los índices de satisfacción por parte de los usuarios frente al proceso, tomado de evaluaciones periódicas realizadas a las familias atendidas.

En un primer momento se describe el método que permitió el desarrollo de la investigación. En un segundo momento, se socializan los resultados a partir de variables como ciclo vital, conformación familiar, motivos de consulta expresados por los usuarios, problemáticas definidas por los 
terapeutas, duración del proceso terapéutico, número de citas, entre otras, relacionándolas con objetivos logrados durante el proceso. En un tercer momento se realiza la discusión de los resultados y las conclusiones del estudio. Se espera que esta investigación aporte información sobre el alcance que tiene la acción terapéutica, motive a fortalecer el quehacer profesional y contribuya a darle significado al ejercicio de la terapia familiar.

Estos hallazgos permiten generar acciones de índole educativa y preventiva acerca de la importancia de acudir a tiempo a un proceso de ayuda, conocer las características de las familias que logran resolver sus dificultades, además de generar esperanza y conciencia en los terapeutas, así como en las familias que atienden y se enfrentan a diversos problemas o conflictos.

\section{Método}

Se trata de un estudio de tipo descriptivo que busca identificar las características socio-familiares y algunos aspectos del proceso terapéutico con consultantes cuyas historias clínicas han logrado los objetivos de intervención propuestos en el Centro de Familia de la Universidad Pontificia Bolivariana. El diseño es primordialmente cuantitativo, es decir, aquel tipo de estudio que permite recoger información sobre conceptos o variables, y cuyos resultados permiten describir el fenómeno de interés. Su objetivo no es indicar cómo se relacionan las variables, sino cómo se presentan y distribuyen diferentes características de la población en estudio.

\section{Muestra}

La fuente primaria para la recolección de la información ha sido la historia clínica del Centro de Familia, en la que cada profesional registra datos sociodemográficos como edad, sexo, parentesco, nivel educativo, profesión, ocupación, procedencia, forma de organización familiar y ciclo vital familiar, número de miembros, datos sobre la intervención recibida como motivo de consulta expresados por los consultantes, periodo o año de atención, número de citas cumplidas y no cumplidas, motivo de finalización y logros 
alcanzados. El universo del estudio lo constituyeron las 4.134 familias y parejas que logran el cumplimiento de los objetivos planteados al iniciar la consulta. En este artículo se presentan los hallazgos relativos a las 2.520 familias consultantes que constituyeron el total de familias con logro de objetivos, las demás historias hacen parte de procesos terapéuticos con parejas que serán objeto de posteriores artículos.

\section{Instrumentos}

El procesamiento de datos se llevó a cabo a través del software denominado Sinhamic (sistema de información para la administración y el manejo de historias clínicas), estructurado y aprobado por personal especializado del Centro de Investigación para el Desarrollo de la Investigación y la Innovación (CIDI) y del Centro de Familia de la UPB, registrado ante el Ministerio del Interior y de Justicia, y en la Dirección Nacional de Derechos de Autor; es función de los profesionales mantenerlo actualizado con la información que se genere en el transcurso de cada sesión terapéutica.

\section{Procedimiento}

Los datos fueron exportados a una base en formato Excel, y procesada en el Statistical Package for the Social Sciences (SPSS), versión 22, licenciado por la UPB, el cual posibilitó la obtención de todas las tablas descriptivas que permitieron dar cuenta de los objetivos.

\section{Resultados}

Este apartado da cuenta de los hallazgos obtenidos y las cifras encontradas que permiten formular una respuesta a los objetivos de la investigación a partir de descripciones y gráficos sobre algunas características de los consultantes, de las familias, de las problemáticas atendidas y del proceso terapéutico. 
En cuanto al origen de la población atendida, corresponde en un 52,1\% a personas con categoría de afiliados, esto significa que son consultantes que provienen de diferentes convenios que tiene el Centro de Familia con instituciones, empresas y cajas de compensación familiar, entre otras; el $43,8 \%$ son beneficiarios, es decir, personas que derivan sus servicios de quien en primera instancia tiene el derecho a recibir el servicio, pueden ser cónyuges, hijos, hermanos, padres, entre otros; por último, el 4,0\% está representado por la consulta particular. Estas características de la población atendida permiten afirmar que los servicios del Centro de Familia favorecen a un sector de la población de Medellín y su área metropolitana que se caracteriza como trabajador y asalariado. La siguiente figura ilustra acerca de las formas de organización familiar de los consultantes que logran los objetivos terapéuticos en el Centro de Familia de la UPB (figura 1).

\section{Figura 6. Formas de organización familiar}

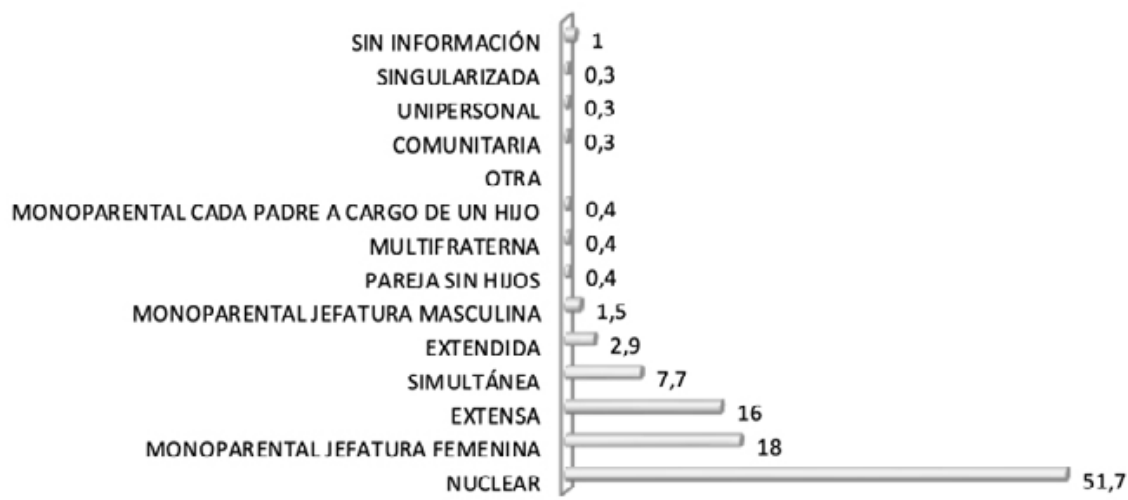

Fuente: elaboración propia.

Si bien predomina la familia de conformación nuclear, se aprecia que también figuran otras que dan cuenta de los cambios que está viviendo esta institución social, lo cual hace que exista la posibilidad de atender familias de corte tradicional, como aquellas con otros parientes, o con un solo padre, hasta 
llegar a los reconocidos como los míos, los tuyos y los nuestros, o incluso grupos fraternos, es decir, de hermanos solos.

Entre nuestros hallazgos, la familia nuclear continúa sobresaliendo como la forma predominante con un puntaje de más de un $50 \%$ de la población que termina procesos con objetivos cumplidos, lo que equivale a resaltar que dicha organización familiar no está exenta de vivir situaciones que los lleven a consultar y la participación en terapia les posibilite encontrar soluciones y alcanzar unas mejores relaciones. Sobresalen también las familias monoparentales con jefatura femenina con un $18 \%$, las familias extensas con un $16 \%$, y otras como las de padres que después de una separación vuelven a conformar pareja, denominadas familias simultáneas, con un 7,7 $\%$, entre aquellas más significativas que consiguieron resolver situaciones problemáticas y recuperar la armonía familiar.

Tal diversidad de organizaciones familiares es descrita por Pliego (2013), al formular en su estudio que en el pasado histórico reciente, el patrón cultural de mayor frecuencia fueron las familias nucleares o que residían con otros parientes, es decir, familias extensas. Sin embargo, en la actualidad se observa una disminución constante de su prioridad demográfica junto al desarrollo cada vez mayor de otros arreglos sociales como el de padres que se han divorciado o separado y se han casado en dos o más ocasiones, hijos con papás o mamás solos, parejas que no tienen hijos y no planean tenerlos; así como otras situaciones más (p.13). En la mayor parte de los países que incluye el estudio (Australia, Brasil, Canadá, Chile, Colombia, España, Estados Unidos, Holanda, Japón, México, Noruega, Perú, Reino Unido) los cambios son de tal magnitud que las consecuencias resultan por demás significativas.

Es importante señalar que el Centro de Familia, como institución prestadora de servicios en salud mental perteneciente a una universidad católica, no excluye ninguna forma de organización familiar en la atención que ofrece, y en él tienen cabida todas las configuraciones familiares que se han ido creando en respuesta a las exigencias sociales, económicas, de sobrevivencia y relacionales. 
Otra de las variables de análisis que se retoma para el estudio de las características de las familias que logran los objetivos terapéuticos es el ciclo vital familiar. A continuación, la figura 2 ilustra lo encontrado.

\section{Figura 7. Ciclo vital}

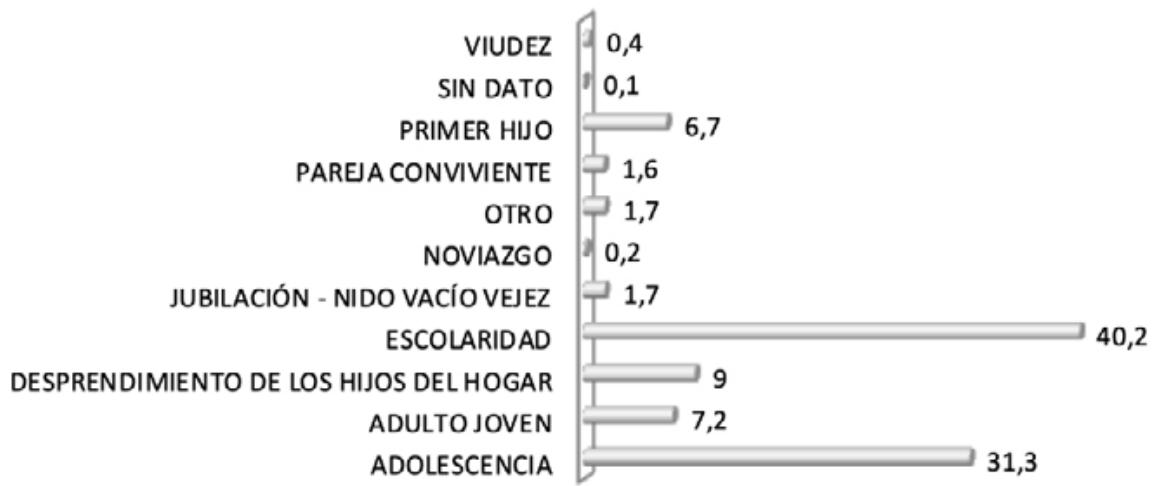

Fuente: elaboración propia.

Las etapas del ciclo vital, también llamadas transiciones familiares, según enuncian Zapata y Agudelo (2015):

Hacen referencia a los recorridos vividos e identificados por las mismas familias, y las consecuentes contradicciones y complejidades que pueden tener en tanto su vida recrea la discontinuidad y la fisura, ejemplo de ello: estar hoy de una manera y mañana de otra, construir una nueva historia cada tanto, ver salir a los hijos prematuramente por la interrupción de una moratoria social ideal que poco aplica para nuestros niños y jóvenes, familias que deben contener a sus miembros por la precariedad de las condiciones en la vida diaria, en la que resulta casi impensable la posibilidad de "vaciar" el nido, dado que el destete no se da de los hijos a los padres sino a la inversa, pues son aquellos los que muchas veces resuelven el sustento de los hogares (p. 21). 
Podríamos identificar en la figura que quienes consultan en familia están ubicados en el ciclo de la escolaridad de los hijos y en la adolescencia. Al comparar estos resultados con la investigación también realizada en el Centro de Familia de la Universidad Pontificia Bolivariana por Agudelo, Bernal, Estrada, Palacio y Posada (2013) sobre problemáticas familiares, encontramos que los ciclos relacionados con los procesos de crecimiento y desarrollo de los hijos son los que más demandan la búsqueda de intervención terapéutica, la atención profesional se convierte en un factor protector y de beneficio para los miembros de la familia que posibilita atender a tiempo las tensiones y dificultades propias de la etapa que están viviendo, evitando así que se conviertan en problemáticas más complejas que requieran una intervención mayor.

Llama la atención además, al revisar la tabla estadística del ciclo vital en la investigación antes citada de Agudelo et al. (2013), cómo los momentos que predominan en la transición familiar que los llevan a buscar ayuda son los siguientes: el adulto joven, adolescencia, escolaridad, desprendimiento de los hijos del hogar y nacimiento del primer hijo; estos mismos momentos se encuentran en la presente investigación, solo que consignados en distinto orden, logrando a su vez también ser reportados al final como procesos que han permitido el logro de los objetivos terapéuticos.

Con relación a las problemáticas por las cuales consultan las familias que logran los objetivos terapéuticos el Centro de Familia de la UPB, se registra la figura 3. 
Figura 8. Problemáticas por las cuales consultan las familias

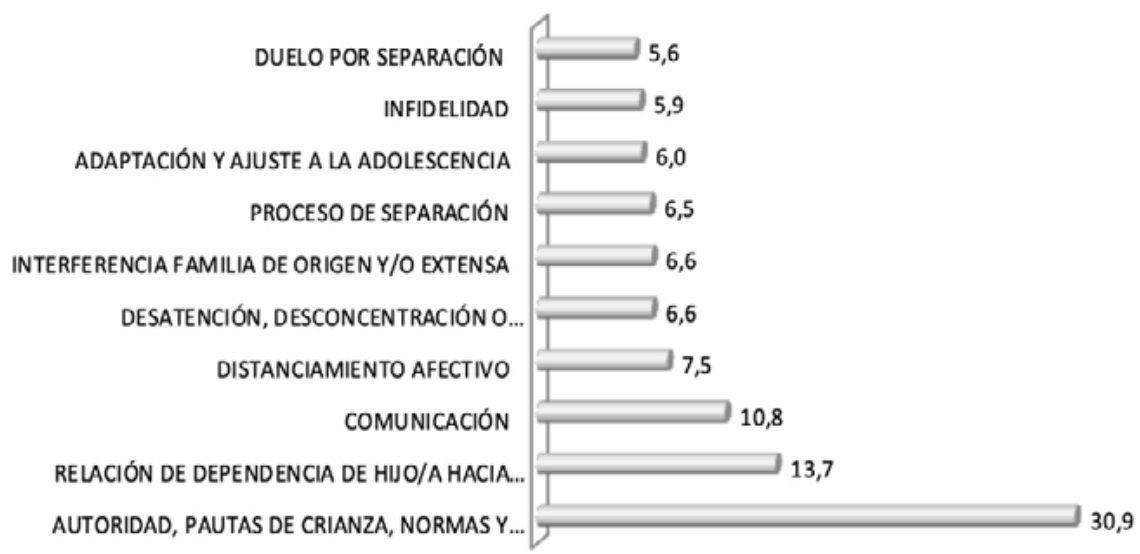

Fuente: elaboración propia.

En el estudio Problemáticas de pareja y familia en consultantes del Centro de Familia de la UPB realizado por Agudelo et al. (2013) se entiende el concepto de problemática como:

Una situación relacional de familia o pareja que genera tensión, preocupación, sufrimiento, conflicto, malestar y alerta emocionalmente a cada uno de los implicados y a todos en sus interacciones. Algunas parejas y familias acuden a terapia al sentir que no han logrado manejar y resolver dicha situación y por lo tanto requieren ayuda de otros sistemas externos. Otros pueden buscar la opción de terapia acogiendo la indicación o sugerencia de alguien que connota su situación como problemática y que por lo tanto requiere ayuda especializada como es el caso de un terapeuta (p. 27).

La problemática, así entendida, se concibe no como algo circunstancial y pasajero sino como una condición constante que involucra a todo un subsistema, sea conyugal, parental o fraterno, y que puede ser transformada para alcanzar formas de funcionamiento más estables. 
Las problemáticas por las cuales más consultan las familias registradas en las historias clínicas, según el criterio del profesional, señalan como puntos principales la autoridad y pautas de crianza, normas y sanciones, la relación de dependencia del hijo hacia el padre, madre y viceversa, y los problemas de comunicación. Los profesionales tienen la posibilidad de fomentar diálogos tendientes a favorecer nuevos relatos, encontrar posibilidades y generar nuevas formas de acción más efectivas en su rol parental. Otras dificultades, en menor porcentaje, que también se lograron resolver positivamente son: el distanciamiento afectivo, desatención, desconcentración y comportamiento perturbador en clase por parte del hijo, interferencia de la familia de origen o extensa, procesos de separación, entre otros.

La atención oportuna y la actitud colaborativa de los padres como responsables del direccionamiento familiar en estas etapas de escolaridad y adolescencia, con la participación de los hijos menores, adolescentes, adultos y otros miembros de la familia que han hecho parte de los procesos terapéuticos, se constituyen en factores que favorecen, alientan y promueven el cambio de las crisis del desarrollo o vitales, así como otras de carácter más problemático.

Ahora bien, una categoría que resulta novedosa en el estudio, que no ha sido analizada en otras investigaciones de acuerdo a nuestra revisión de antecedentes, es el motivo de consulta expresado por los usuarios. Utilizando el lenguaje del consultante tal como lo expresaron Anderson y Goolishian (1996), "el cliente es el experto en narrar sus dificultades tal como él las vive" (p. 46).

De la totalidad de los registros de motivos de consulta se extraen algunas narrativas que reflejan dolor, sufrimiento, amargura, y pesadumbre de los consultantes e ilustran con claridad las situaciones que motivan a las personas a asistir a terapia. Veamos algunos ejemplos:

Tenemos muchos problemas, no nos entendemos para manejar el hijo: ella es muy estricta, yo soy más flexible, discutimos mucho eso; cuando ella castiga al niño yo intervengo porque me parece que es muy drástica, yo manejo al niño diferente.

Mi hija menor es grosera, agresiva, desobediente, brusca con los compañeros. Yo me irrito, me desespero, le pego; el padre 
es ausente por condición laboral; hace tres meses hay cambios en la vida escolar, familiar y social.

Estamos preocupados porque nos inquieta la hija mayor, se mantiene estresada, agresiva, se siente grande, es irritable y entre toda la familia queremos ayudarle. Ella se desarrolló muy rápido, el cambio fue muy brusco.

Mi madre me abandonó desde que estaba pequeña, fui criada por mi padrastro, quien murió hace unos meses. Tengo dos hijos, fui madre a los 15 años y no deseaba tenerlos. Mi hijo mayor es retraído, no presta atención.

A la casa llegó hace cinco o seis meses un joven a hacer un trabajo, todo tatuado, amante de la música metálica, que contaba tenía una vida desorganizada y ahora está con mi hija, quien está en embarazo de él y hemos tenido muchos problemas y desacuerdos por esta relación.

Me siento muy anulada en la casa, yo soy la que tengo que hacer todo en la casa, encargarme de los hijos, y Jaime en lo suyo, con su libertad. Estuvimos ocho años siempre juntos parrandeando y paseando, pero desde que nacieron los hijos todo es distinto, yo ya no puedo por los hijos, y Jaime dice que eso es percepción mía.

Los problemas de nosotros tienen que ver con la familia de ella que se meten en las relaciones de nosotros, opinan y toman decisiones sin mí y ella lo acepta. Está muy metida en la casa de la mamá y la mamá en la mía. Además, me preocupa mucho que el hermano de ella es alcohólico y drogadicto y esto puede afectar a nuestro hijo.

Tuvimos un incidente con Juliana, nuestra hija mayor, ella se tomó unas pastillas psiquiátricas que le regaló una amiga del colegio que la intoxicaron y la tuvimos que llevar a la clínica, también se está haciendo cortes en los brazos y va perdiendo el año. 
En algunos de los relatos expresados en la voz de las familias, se encontraron situaciones relacionadas con la descalificación e incoherencia de los padres en el ejercicio de la autoridad, el comportamiento de los hijos, especialmente adolescentes, descritos por los padres como "desafiantes, irritables y agresivos". En otros casos se presentan adolescentes con limitaciones para asumir la maternidad. Estas narrativas, notablemente perturbadoras, dolorosas y complejas según los hallazgos y el cierre del proceso realizado por los profesionales en los registros, logran ser superadas y se alcanzan los objetivos terapéuticos, lo que atribuye a la terapia un panorama alentador frente a los dilemas que presenta la convivencia, el crecimiento, el paso por las etapas vitales y los devenires de la vida.

Se informa a continuación el tiempo de duración en meses de los procesos terapéuticos de las familias que logran objetivos (figura 9).

\section{Figura 9. Tiempo del proceso en meses}

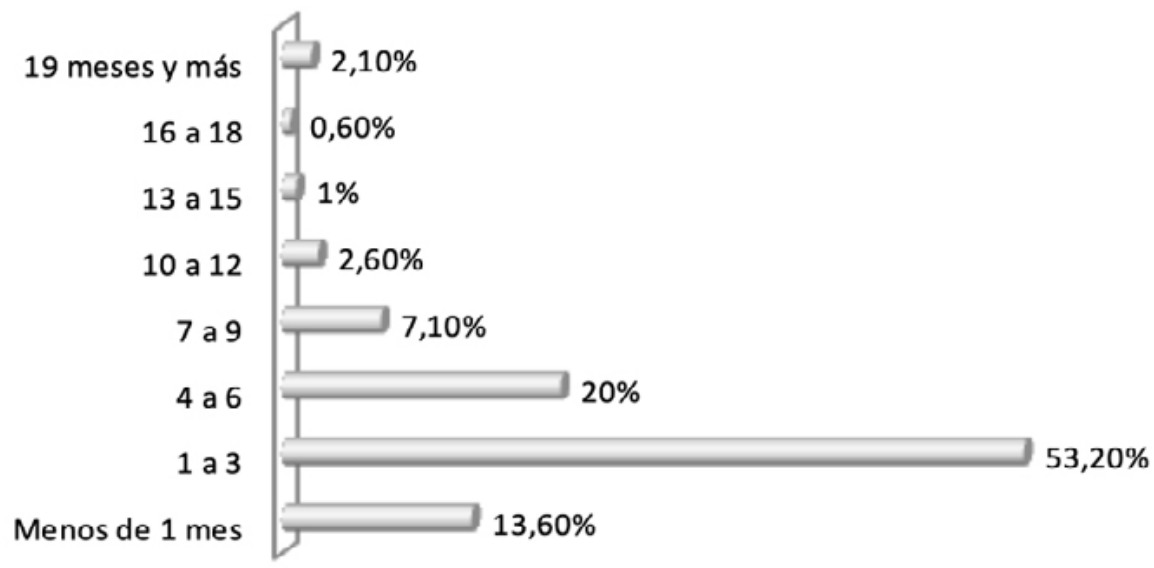

Fuente: elaboración propia.

El mayor porcentaje de las familias, $53,2 \%$, consulta por periodos que comprenden de uno a tres meses, continúa, con un $20 \%$, aquellas que participan del proceso durante cuatro a seis meses y, finalmente, $13,6 \%$ que asiste menos de un mes, como tiempos significativos que corresponden a 
los criterios de la terapia breve. En cuanto al número de citas, el porcentaje más significativo es de cinco o más, con un 70,8 \%, mientras que el 26,1\% asiste a entre dos, tres y cuatro citas, tal y como lo ilustra la figura 5.

Figura 10. Número de citas por proceso

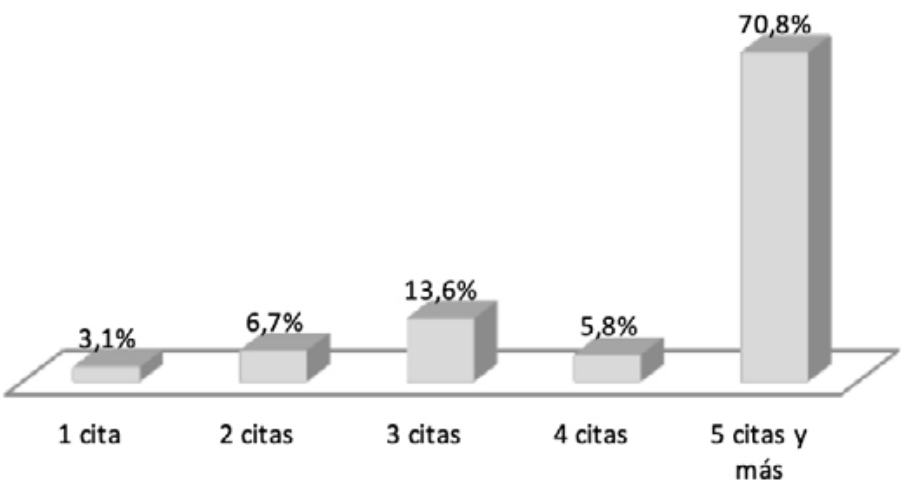

Fuente: elaboración propia.

La terapia "breve" es entendida como un proceso cuya duración oscila entre una y diez citas, la cual suele durar una hora y se asigna de manera más separada a medida que se producen mejorías en la situación que plantean los consultantes. De este modo, se suelen dejar una o dos semanas entre la primera entrevista y la segunda, aumentando posteriormente el intervalo a tres semanas o un mes. Por lo tanto, una terapia de seis sesiones ocupa por lo general entre dos y seis meses.

Otras experiencias, según se puede leer en Rodríguez-Arias, Otero, Venero y Ciordia (2004), revelan:

Que los casos de éxito se caracterizan por quejas de inicio más reciente, que no han tenido tratamientos previos (...). En estos casos las sesiones son más cortas; pero no más frecuentes, y la mejoría empieza tras la primera sesión. Según lo esperado, ni las sesiones ni los tratamientos en su conjunto son más largos; es 
decir, se fracasa en los mismos tiempos en los que se resuelve (...). No se confirman diferencias (...) ni en el nivel educativo ni en el formato individual o familiar; pero sí se tiende a tener más éxito con los grupos de población más jóvenes (p. 5).

En este sentido, otra experiencia similar surge de una investigación realizada por la Clínica de Terapia Familiar del Hospital San Vicente (Vásquez et al., 1987), donde el mayor porcentaje (30\%) de quienes terminan el proceso lo hacen en un tiempo que va de 19 a 24 meses; sin embargo, hay un $40 \%$ de quienes culminan el tratamiento que lo hacen en menos de 12 meses, igualmente un $30 \%$ suspenden el acompañamiento terapéutico antes de cumplir un año de consulta.

\section{Grado de satisfacción de los usuarios del Centro de Familia}

En el trascurso del proceso terapéutico la percepción de los usuarios sobre el grado de satisfacción frente al servicio -medido a través de una encuesta diligenciada por los consultantes que llevan más de tres citas, desde 2008, periodo en el cual se empiezan a implementar políticas de calidad del servicio, hasta la fecha, con un total de 4.469 encuestas respondidas- es de 4,8 en una escala de uno a cinco. Este hallazgo corresponde a los procesos de calidad de la institución realizados semestralmente y permite pensar que constituye un factor importante a ser revisado debido a que los niveles de satisfacción también pueden tener incidencia con la adherencia al proceso y, por ende, al logro de los objetivos.

\section{Discusión}

Es de destacar cómo en el estudio objeto de este artículo y en otros realizados en el Centro de Familia de la UPB sobre la caracterización de las familias usuarias de los servicios, así como en el llevado a cabo por Vásquez et al. (1987), que da cuenta de las características familiares de quienes 
(1)

consultaron en la Clínica de Terapia Familiar de la Facultad de Medicina de la Universidad de Antioquia, las conformaciones familiares no han variado, en estos estudios se evidencia que aquellas que más consultan son la nuclear, la monoparental femenina y la extensa. La única diferencia es que para la época de los años 80 en que fue realizada la investigación de Vásquez et al. (1987), no se identifica la familia simultánea (los tuyos, los míos y los nuestros) que en la posmodernidad se presenta con mayor frecuencia y es reportada en el presente estudio.

Es importante subrayar cómo estos grupos familiares, con sus conformaciones diversas, se movilizan a consultar; se destaca en ellos la conciencia por buscar asesoría profesional, no permanecer paralizados ante los problemas y asumir una alternativa o decisión que no es fácil de tomar ya que implica exponer sus dificultades a un tercero. La otra opción que pueden tomar es la de continuar resolviéndolos con sus intuiciones o experiencias familiares previas; no obstante, acuden en busca de un proceso terapéutico que les permita modificar y encontrar nuevos significados a las situaciones difíciles que están viviendo.

Las familias se movilizan a consultar en aquellas etapas del desarrollo que más riesgos pueden traer a los hijos y al sistema familiar, teniendo en cuenta las nuevas experiencias producto del crecimiento y el desarrollo que podrían ser las que activan a los padres a buscar bienestar. Se presentan, en orden de prioridad, los momentos vitales que llevan a la consulta, como las crisis propias de las etapas, escolar, adolescencia, desprendimiento de los hijos del hogar, hijos adultos y el nacimiento de los hijos, logrando en ellas solución a sus inquietudes y dificultades relacionales. Es significativo que las necesidades propias de la pareja en las distintas etapas del desarrollo, como la de ajuste al matrimonio, jubilación o viudez, sean de menor demanda de atención, lo que puede explicarse con el hecho de que los hijos movilizan con mayor énfasis la ayuda para el sistema familiar.

Según señala Cuervo (2010), la prevención de factores de riesgo en la familia que influyen negativamente en los estilos de crianza y en el desarrollo socioafectivo durante la infancia es una responsabilidad de los distintos profesionales y sectores para lograr un adecuado desarrollo de habilidades, competencias parentales, habilidades emocionales y conductas prosociales en los niños, niñas y adolescentes (p. 117). En este sentido, la terapia se 
convierte en un factor protector que permite acompañar a los padres en las vicisitudes que se presentan en las etapas de la escolaridad y la adolescencia, promoviendo canales de comunicación claros, asertivos, que se convierten en un eje transversal que aporta a la resolución de otro tipo de dificultades.

Sobre la duración del proceso terapéutico, Rodríguez y Beyerbach (1994) plantean que, aunque el modelo de terapia familiar no varía esencialmente de un caso a otro, los avances en las distintas sesiones están sujetos a la capacidad de cambio de los consultantes, los cuales pueden darse en un promedio de cinco sesiones, aproximadamente de una hora cada una (p. 250), ello depende, además, de los recursos interaccionales con que cuente la familia.

Las familias consultantes que culminaron el proceso con logro de objetivos terapéuticos fueron 2.720 , que equivalen al $20 \%$ del universo total atendido en el Centro de Familia, es decir, 48.759 familias en total. Este alcance es definido por el terapeuta y los consultantes en citas previas a la finalización del proceso. Tal aspecto se constituye en tema de conversación abordado durante la consulta que dará pie a la culminación de la terapia, y al ser contrastado con otras investigaciones sobre procesos terapéuticos -por ejemplo la de Rodríguez-Arias et al. (2004) en el estudio de evaluación de resultados en terapia familiar breve-, referencian, según varios estudios anteriores, el logro de objetivos terapéuticos en el $77 \%$, así como el de un $23 \%$ que no lo hace (p. 4). En seguimiento realizado veinte meses después de la finalización del tratamiento, han conseguido resolver el problema por el que consultaron las dos terceras partes y han recaído un $19 \%$. Además, registran como un dato importante que el $66 \%$ de los contactados en seguimiento informan haber mejorado en áreas no tratadas en las sesiones, es decir, se observa un efecto irradiación.

En la misma dirección, Vásquez et al. (1987) registran en su publicación sobre los cinco años de la clínica de Terapia Familiar del Hospital San Vicente un $30,7 \%$ de mejoría significativa y un 30,7\% de persistencia en el tratamiento familiar. Esto concuerda con los hallazgos de la investigación del Centro de Familia por tratarse posiblemente de familias del mismo contexto social y cultural. Además de ser un universo alto el tenido en cuenta en el presente estudio, los porcentajes de estas investigaciones son significativos en la medida en que reportan alivio al grupo familiar, contribuyendo al mejor cumplimiento de funciones y estabilidad emocional para sus miembros. 
Es importante resaltar como característica del tratamiento llevado a cabo en el Centro de Familia de la UPB, que este es un proceso de ayuda con una duración de entre uno y seis meses, y una asistencia a consultas de entre cinco y más citas. Este hallazgo permite a los profesionales informar y orientar a los usuarios acerca de este factor de duración que, con frecuencia, quieren conocer si cuentan con la disponibilidad acorde con sus ocupaciones laborales y necesidades económicas. Al mismo tiempo, esto propicia hacer de cada consulta un espacio significativo en el cual el profesional construya con la familia como si fuera la última vez que tiene acceso a interactuar con ella, entendiendo que gran parte de los procesos son de corta duración.

\section{Conclusiones}

La conformación familiar que predomina en la población estudiada es la nuclear, algo que no es de extrañar, pues múltiples estudios apuntan en esa dirección e identifica a esta tipología como la de mayor prevalencia tanto en población general como en aquellas que acuden a consulta. Este hecho demuestra que esta configuración familiar es tan susceptible y vulnerable como las otras que aparecen en los lugares posteriores: monoparental con jefatura femenina, extensa o simultánea.

De acuerdo con la caracterización de las familias consultantes, sobresalen las etapas del ciclo vital como son: escolaridad, adolescencia, desprendimiento de los hijos, adulto joven y nacimiento del primer hijo, sobre las cuales se puede percibir que son las que más tensiones generan dentro de las relaciones y requieren ajustes para posibilitar a la pareja y a la familia espacios y encuentros idóneos que les permitan trascender las inestabilidades, propiciando un mayor desarrollo de sus miembros.

Las problemáticas de mayor consulta por parte de las familias se refieren a situaciones como dificultad en el ejercicio de la autoridad, pautas de crianza, relación de dependencia del hijo/hija, y problemas de comunicación, las cuales se constituyen en el punto de partida para promover cambios en las interacciones del sistema familiar y propiciar la estabilidad de sus miembros. 
Los procesos terapéuticos que logran el alcance de objetivos permiten a los consultantes obtener ideas, explicaciones, nuevos significados que posibilitan el cambio en un intervalo de cinco citas o más llevadas a cabo en un periodo de seis meses. Esta situación se evidencia en las notas de cierre de las historias clínicas por medio de la retroalimentación que los consultantes hacen al terapeuta acerca de sus avances y los alcances que ha tenido la terapia.

A la luz del profesional tratante, se tuvieron en cuenta las historias de las familias que han cumplido con el logro de los objetivos terapéuticos según la perspectiva del profesional, quien da por finalizada la atención de acuerdo con los consultantes. Este estudio no incluye a las familias que terminan la terapia con objetivos parciales a criterio del profesional, con el fin de no contaminar el análisis con otros motivos de finalización, como pueden ser la deserción, el no estar preparado para un proceso terapéutico, la incompatibilidad de horario por estudio o trabajo, o bien la pérdida de afiliación al servicio.

Para el Centro de Familia es significativo, al aplicar la evaluación de satisfacción a los consultantes por medio de un cuestionario virtual o presencial, conocer que los resultados, una vez finalizada la intervención, son positivos y se ven reflejados en la calidad de la atención, las instalaciones físicas, el profesionalismo de su terapeuta tratante y la participación activa de ellos mismos, llegando a afirmar que recomendarían el servicio a sus familiares y amigos.

La investigación en temas de familia, situaciones individuales, grupales o sociales acostumbra a centrarse en las indagaciones que se hacen desde el déficit, las perturbaciones, los diagnósticos o las patologías. La principal novedad de este estudio pasa por dirigir sus resultados a la posibilidad de centrar la mirada en factores alentadores, positivos e identificar los modos de encontrar en la terapia algunas características de las familias y del proceso terapéutico de aquellos que logran resolver sus dificultades.

Los hallazgos constituyen una invitación a fortalecer el quehacer profesional y permiten la realización de una autoevaluación constante por parte de los terapeutas sobre aspectos en los cuales deben fortalecer su atención a los usuarios, identificando con claridad temas como ciclo vital, tipología familiar, motivo de consulta y duración de los procesos terapéuticos, que permiten inferir la viabilidad del logro de objetivos en estas variables. 
Los relatos obtenidos directamente de la consulta sobre lo que para los consultantes es el motivo que los lleva a buscar ayuda, pueden constituirse en indicios que conduzcan a otros profesionales o estudiantes en formación a profundizar en tales dificultades y plantear temas de investigación.

\section{Referencias}

Agudelo, M., Bernal, I. C., Estrada, P., Palacio, M. A. y Posada, F. A. (2013). Problemáticas de pareja y familias en consultantes del Centro de Familia de la Universidad Pontificia Bolivariana. Universidad Pontificia Bolivariana.

Anderson, H. y Goolishian, H. (1996). El experto es el cliente: la ignorancia como enfoque terapéutico. Paidós.

Cuervo, A. (2010). Pautas de crianza y desarrollo socioafectivo en la infancia. Diversitas. Perspectivas en Psicología, 6(1), 111-121. https://bit.ly/3mIdLTs

Pliego, F. (2013). Tipos de familia y bienestar de niños y adultos. El debate cultural del siglo XXI en 13 países democráticos. Universidad Nacional Autónoma de México. https://bit. ly/33PLRMH

Rodríguez-Arias, J. L., Otero, M., Venero, M. y Ciordia, N. (2004). Estudio de evaluación de resultados en terapia familiar breve. Papeles del Psicólogo, 25(87). http://www.papelesdelpsicologo.es/vernumero.asp? id=1136

Rodríguez, A. y Beyerbach, M. (1994). Terapia sistémica breve: trabajando con los recursos de las personas. En M. Garrido y J. García (eds.), Psicoterapia: modelos contemporáneos y aplicaciones (pp. 241-289). Promolibro.

Vásquez, E., Mira, H., Molina, B. M., Franca, M. B., López, O. L. y Giraldo, C. A. (1987). Cinco años de la Clínica de Terapia Familiar. Una evaluación a través de las familias y los terapeutas. En II Encuentro Nacional de Terapia Familiar. Encuentro llevado a cabo en Medellín, Colombia.

Zapata, J. J. y Agudelo M. E. (2015). El recorrido vital familiar en la contemporaneidad. Tesis Psicológica, 10(1), 12-29. https://bit.ly/3ckqMOx 